Supplemental Material for

\title{
Molecular Analysis of the Benastatin Biosynthetic Pathway and Genetic Engineering of Altered Fatty Acid - Polyketide Hybrids
}

Zhongli Xu, Angéla Schenk \& Christian Hertweck*

Contribution from the Leibniz. Institute for Natural Product Research and Infection Biology, HKI, Dept. of Biomolecular Chemistry, Beutenbergstr. 11a, 07745 Jena, Germany, and the Friedrich-Schiller-University, Jena, Germany

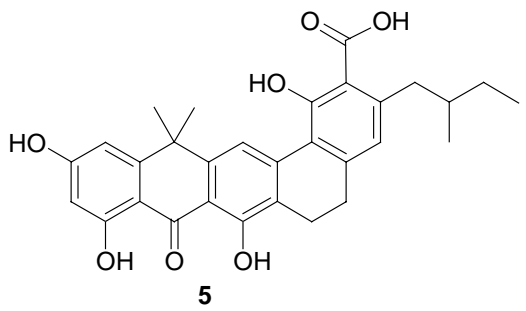

Figure S1: Tentative structure of 5 .

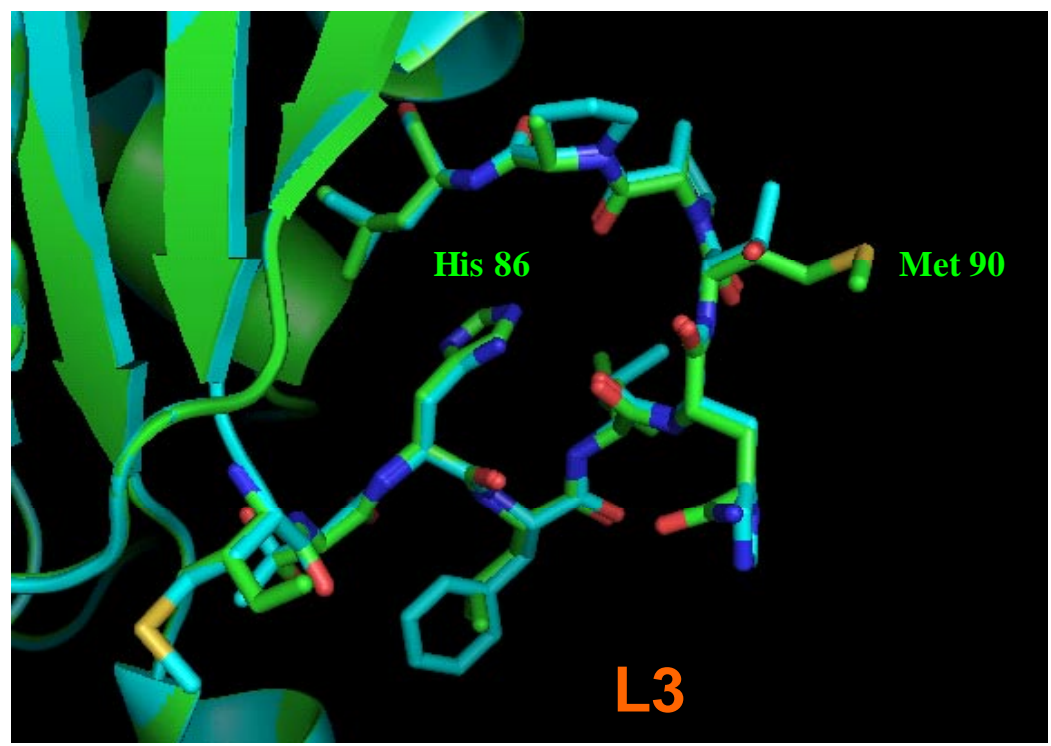

Figure S2: Superposed putative binding pocket (Loop 3) of BenQ (green) and that of ZhuH (cyan). The protrudent amino acid residues His86 and Met90' (from its counterpart of the dimer) of BenQ allowed only suitable sized straight-chain fatty acid to be accommodated in this substrate binding pocket. (Molecules visualized through PyMOL. 